\title{
Exponential Stability and Availability Analysis of a Complex Standby System
}

\author{
Chunli WANG \\ Institute of Information Technology, University of Electronic Technology, Guilin 541004, China \\ Mingyan TENG \\ Department of Mathematics, Bohai University, Jinzhou 121002, China
}

Fu ZHENG

Department of Mathematics, Bohai University, Jinzhou 121002, China

E-mail: fz555888@aliyun.com

\begin{abstract}
In this paper, we first investigate the solution of the two correlated units redundant system with two types of failure. By using the method of functional analysis, especially, the $c_{0}$ semigroup theory of bounded linear operators on Banach space, we prove the well-posedness and the existence of positive solution of the system. By analyzing the spectra distribution of the system operator, we prove that the dynamic solution of the system asymptotically converges to the nonnegative steady-state solution which is the eigenfunction corresponding to eigenvalue 0 of the system operator. Furthermore, we discuss the exponential stability of the system. Finally, we analyze the reliability the system with the help of our main results and present some reliability indices of the system at the end of the paper.
\end{abstract}

Keywords redundant system; $c_{0}$ semigroups; well-posedness; stability; availability

\section{Introduction}

Reliability is an important concept of various complex systems. The need of obtaining highly reliable system has became more and more important with the development of the modern technology. The high degree of reliability is usually achieved by introducing redundancy. The redundant system discussed here comes from [1]. It consists of two sub-systems A and B, connected in parallel having one regular and one expert repairman. This system has four states, viz. normal, degraded, failed and waiting. The sub-system B is in standby mode and consists of further two units, viz. $\mathrm{B}_{1}$ and $\mathrm{B}_{2}$, connected in parallel. It is assumed that when both the units of sub-system B start functioning simultaneously, then sub-system B works like the sub-system A. So, when some failure occurs in sub-system A, the two units of sub-system $\mathrm{B}$ will be in operable condition through perfect switching over device. If any one unit of subsystem B fails (after failing sub-system A), the system will work but in degraded stage. The system will be in down state only when both sub-system A and B fail or due to occurrence of environmental failure.

Received October 24, 2013, accepted March 6, 2014

Supported by the National Natural Science Foundation of China (Grant No. 11201037, 11371070) 
In [1], the well-posedness and asymptotic stability of the redundant system have been obtained. Because it is difficult to determine the convergence rate of the dynamic solution only by asymptotic stability, the study of the exponential stability is in demand, which is a more valuable property in application. We will continue to discuss the above system and obtain the exponential stability of the system. It should be pointed out that, in recent years, though there are several papers (e.g. see [3-6]) on the exponential stability of the repairable systems, the method of [2-5] is involved in quasi-compactness and is hard to be applied here. Moreover, as the applications of the main results of [1], we will calculate the stationary availability of the system with another method different from the traditional one [6-9].

The rest of this paper is organized as follows. In section 2, we introduce the mathematical model and some notations of the system. In the meantime, some results on the well-posedness and stability are introduced in this section. In section 3, the exponential of the system is presented. In the last section, we investigate the stationary availability of the system with another method different from the traditional one and give several numerical simulation examples.

\section{Mathematical model formulation and some results}

Most materials of this section come from [1] and for convenience, we do not restate and introduce them freely. Let $a=\lambda_{0}+\lambda_{1}+\lambda_{2}, p_{0}(t)$ be the probability that the system is in operable state at time $t, p_{1}(t)$ probability that the system is in waiting state at time $t, p_{i}(t, x) \mathrm{d} x$ probability that the system is in operable state at time and elapsed repair time lies between $x$ and $x+\mathrm{d} x$, where $j=8,9,10,11$, and $p_{i}(t, x) \mathrm{d} x$ probability that the system is in failed state at time $t$ and elapsed repair time lies between $x$ and $x+\mathrm{d} x$, where $i=7,12$. With some additional assumptions, the differential equations of the system are obtained,

$$
\begin{gathered}
p_{0}(t)=\int_{0}^{\infty} p_{2}(t, x) \mu_{1}(x) \mathrm{d} x+\int_{0}^{\infty} p_{3}(t, x) \mu_{2}(x) \mathrm{d} x+\int_{0}^{\infty} p_{4}(t, x) \mu(x) \mathrm{d} x \\
+\int_{0}^{\infty} \eta(x)\left[p_{5}(t, x)+p_{6}(t, x)\right] \mathrm{d} x+\int_{0}^{\infty} p_{7}(t, x) \mu_{3}(x) \mathrm{d} x+\int_{0}^{\infty} p_{12}(t, x) \beta(x) \mathrm{d} x \\
{\left[\frac{\mathrm{d}}{\mathrm{d} t}+r_{1}\right] p_{1}(t)=\lambda_{0} \int_{0}^{\infty} p_{4}(t, x) \mathrm{d} x} \\
{\left[\frac{\partial}{\partial t}+\frac{\partial}{\partial x}+\mu_{1}(x)+\lambda_{2}\right] p_{2}(t, x)=0} \\
{\left[\frac{\partial}{\partial t}+\frac{\partial}{\partial x}+\mu_{2}(x)+\lambda_{1}\right] p_{3}(t, x)=0} \\
{\left[\frac{\partial}{\partial t}+\frac{\partial}{\partial x}+\mu(x)+\lambda\right] p_{4}(t, x)=0} \\
{\left[\frac{\partial}{\partial t}+\frac{\partial}{\partial x}+\eta(x)+\lambda_{1}+\lambda_{2}+r_{2}\right] p_{5}(t, x)=0} \\
{\left[\frac{\partial}{\partial t}+\frac{\partial}{\partial x}+\eta(x)+\lambda_{1}+\lambda_{2}\right] p_{6}(t, x)=0} \\
{\left[\frac{\partial}{\partial t}+\frac{\partial}{\partial x}+\mu_{3}(x)\right] p_{7}(t, x)=0}
\end{gathered}
$$




$$
\begin{gathered}
{\left[\frac{\partial}{\partial t}+\frac{\partial}{\partial x}+\mu_{1}(x)+r_{2}\right] p_{8}(t, x)=0} \\
{\left[\frac{\partial}{\partial t}+\frac{\partial}{\partial x}+\mu_{2}(x)+r_{2}\right] p_{9}(t, x)=0} \\
{\left[\frac{\partial}{\partial t}+\frac{\partial}{\partial x}+\mu_{1}(x)+\lambda_{2}\right] p_{10}(t, x)=0} \\
{\left[\frac{\partial}{\partial t}+\frac{\partial}{\partial x}+\mu_{2}(x)+\lambda_{1}\right] p_{11}(t, x)=0} \\
{\left[\frac{\partial}{\partial t}+\frac{\partial}{\partial x}+\beta(x)\right] p_{12}(t, x)=0}
\end{gathered}
$$

and with the boundary conditions

$$
\begin{aligned}
p_{2}(t, 0) & =\lambda_{1} p_{0}(t), \quad p_{3}(t, 0)=\lambda_{2} p_{0}(t) \\
p_{4}(t, 0) & =\lambda_{2} \int_{0}^{\infty} p_{2}(t, x) \mathrm{d} x+\lambda_{1} \int_{0}^{\infty} p_{3}(t, x) \mathrm{d} x \\
p_{5}(t, 0) & =\lambda_{0} p_{0}(t)+r_{1} p_{1}(t)+\int_{0}^{\infty} \mu_{1}(x) p_{8}(t, x) \mathrm{d} x+\int_{0}^{\infty} \mu_{2}(x) p_{9}(t, x) \mathrm{d} x \\
p_{6}(t, 0) & =r_{2} \int_{0}^{\infty} p_{5}(t, x) \mathrm{d} x+\int_{0}^{\infty} \mu_{1}(x) p_{10}(t, x) \mathrm{d} x+\int_{0}^{\infty} \mu_{2}(x) p_{11}(t, x) \mathrm{d} x \\
p_{7}(t, 0) & =\lambda_{3} p_{0}(t), p_{8}(t, 0)=\lambda_{1} \int_{0}^{\infty} p_{5}(t, x) \mathrm{d} x, p_{9}(t, 0)=\lambda_{1} \int_{0}^{\infty} p_{5}(t, x) \mathrm{d} x \\
p_{10}(t, 0) & =\lambda_{1} \int_{0}^{\infty} p_{6}(t, x) \mathrm{d} x+r_{2} \int_{0}^{\infty} p_{8}(t, x) \mathrm{d} x \\
p_{11}(t, 0) & =\lambda_{2} \int_{0}^{\infty} p_{6}(t, x) \mathrm{d} x+r_{2} \int_{0}^{\infty} p_{9}(t, x) \mathrm{d} x \\
p_{12}(t, 0) & =\lambda_{2} \int_{0}^{\infty} p_{10}(t, x) \mathrm{d} x+\lambda_{1} \int_{0}^{\infty} p_{11}(t, x) \mathrm{d} x
\end{aligned}
$$

and the initial conditions $p_{0}=1 ; p_{1}=p_{1}(0, x)=\cdots=p_{2}(0, x)=0$. You can refer to [1] for the concrete meanings of the symbols, such as $\lambda_{0}$ and $r_{1}$ etc.

The equations $(1) \sim(21)$ can be rewritten as an the abstract Cauchy problem (see [11-12]) in Banach space $X$ :

$$
(A C P) \quad\left\{\begin{array}{l}
\frac{\mathrm{d} p(t)}{\mathrm{d} t}=\mathcal{A} p(t), \quad t \geq 0 \\
p(0)=(1,0, \cdots, 0)^{\mathrm{T}}
\end{array}\right.
$$

in which $\mathcal{A}$ is defined in [4] and $X=\mathbb{R}^{2} \times\left(L^{1}\left(\mathbb{R}^{+}\right)\right)^{11}$ is Banach space. For $p=\left(p_{0}, p_{1}, p_{2}(x), \cdots\right.$, $\left.p_{12}(x)\right) \in X$, The norm of $p$ is given by

$$
\|p\|=\left|p_{0}\right|+\left|p_{1}\right|+\sum_{i=2}^{12} \int_{0}^{\infty}\left|p_{i}(x)\right| \mathrm{d} x .
$$

Let $\mu_{4}(x)=\mu(x), \mu_{5}(x)=\beta(x), \mu_{6}(x)=\eta(x)$. The main results of this paper are obtained under the following assumptions.

\section{General Assumptions:}


There exist positive constants $H$ and $c$, such that for any $t \geq 0$,

$$
\frac{1}{t} \int_{t}^{\infty} \mathrm{e}^{-\int_{0}^{u} \mu_{i}(s) \mathrm{d} s} \mathrm{~d} u>H
$$

and

$$
c=\min \left\{\inf _{x \in \mathbb{R}^{+}} \mu_{i}(x), i=1,2, \cdots, 6\right\} .
$$

The physical meaning of the general assumption is the mean repair rate is not too small. By the general assumptions, it is easily to obtain the following identify

$$
\int_{0}^{\infty} u_{i}(x) \mathrm{e}^{-\int_{0}^{x} u_{i}(\tau) \mathrm{d}(\tau)} \mathrm{d} x=1 .
$$

The following theorems are main results of [4].

Theorem $1 \mathcal{A}$ generates a positive $C_{0}$ contractive semigroup $T(t)$ on $X$ and the unique time dependent positive solution to the equations (1) $(21)$ exists.

Theorem 2 Zero is an algebraically simple eigenvalue of $\mathcal{A}$ and the system is asymtotically stable. The steady-state solution is the positive unit eigenfunction corresponding eigenvalue 0 of the generator $\mathcal{A}$.

\section{The exponential stability of the system}

Let $T(t)$ be the semigroups generated by the operator $\mathcal{A}$ and $p(0)$ the initial value, the asymptotic stability shows that

$$
T(t) p(0)=(p(0), Q) \widetilde{p}+T_{1}(t) p(0)=\widetilde{p}+T_{1}(t) p(0), \lim _{t \rightarrow \infty} T_{1}(t) p(0)=0,
$$

in which $\widetilde{p}$ is the positive unit eigenfunction corresponding to eigenvalue 0 of $\mathcal{A}$ and $Q=$ $(1, \cdots, 1) \in X^{*}$.

In general, the above stability does not implies the exponential stability, that is to say there exist positive $\omega$ and $M$ such that

$$
\left\|T_{1}(t) p(0)\right\| \leq M \mathrm{e}^{-\omega t}, \quad t \geq 0 .
$$

But in some special cases this implication holds. Now we will show the exponential stability of the system. First, we introduce some notations and results which come from references [3] and [14].

Let $X$ be a Banach space and $T(t)$ be a $c_{0}$ semigroups on $X$ and $\mathcal{A}$ its generator, for $\lambda \in \rho(\mathcal{A})$, then we have the symbols

$$
\omega_{1}(T)=\lim _{t \rightarrow+\infty} \frac{\ln \|T(t) R(\lambda, \mathcal{A})\|}{t}, s_{0}(\mathcal{A})=\sup \left\{\omega>s(\mathcal{A}): \sup _{\operatorname{Re} \lambda=\omega}\|R(\lambda, \mathcal{A})\|<+\infty\right\},
$$

in which $\omega_{1}(T)$ is the growth bound of the semigroups $T(t)$ and $s_{0}(\mathcal{A})$ is abscissa of uniform boundedness of the resolvent of $\mathcal{A}$. Their relationship is given by the following lemma which comes from [3].

Lemma 1 Let $T(t)$ be a $c_{0}$ semigroup on Banach space $X$ and $\mathcal{A}$ its generator, then $\omega_{1}(T)<s_{0}(\mathcal{A})$.

The following Lemma comes from [14]. 
Lemma 2 For each $y \in X, b \in \mathbb{R}$ and $i b \in \mathbb{C}$, the unique solution to the equations $(i b-\mathcal{A}) p=y$ exists and $p$ is given by

$$
\begin{aligned}
& (a+i b) p_{0}=\int_{0}^{\infty} p_{2}(x) \mu_{1}(x) \mathrm{d} x+\int_{0}^{\infty} p_{3}(x) \mu_{2}(x) \mathrm{d} x+\int_{0}^{\infty} p_{4}(x) \mu(x) \mathrm{d} x \\
& +\int_{0}^{\infty} \eta(x)\left[p_{5}(x)+p_{6}(x)\right] \mathrm{d} x+\int_{0}^{\infty} p_{7}(x) \mu_{3}(x) \mathrm{d} x+y_{0} \\
& \left(r_{1}+i b\right) p_{1}=\lambda_{0} \int_{0}^{\infty} p_{4}(x) \mathrm{d} x+y_{1} \\
& p_{2}(x)=p_{2}(0) \mathrm{e}^{-\int_{0}^{x}\left[i b+\lambda_{2}+\mu_{1}(s)\right] \mathrm{d} s}+\int_{0}^{\infty} \mathrm{e}^{-\int_{\tau}^{x}\left[i b+\lambda_{2}+\mu_{1}(s)\right] \mathrm{d} s} y_{2}(\tau) \mathrm{d}(\tau) \\
& p_{3}(x)=p_{3}(0) \mathrm{e}^{-\int_{0}^{x}\left[i b+\lambda_{1}+\mu_{2}(s)\right] \mathrm{d} s}+\int_{0}^{\infty} \mathrm{e}^{-\int_{\tau}^{x}\left[i b+\lambda_{1}+\mu_{2}(s)\right] \mathrm{d} s} y_{3}(\tau) \mathrm{d}(\tau) \\
& p_{4}(x)=p_{4}(0) \mathrm{e}^{-\int_{0}^{x}\left[i b+\lambda_{0}+\mu(s)\right] \mathrm{d} s}+\int_{0}^{\infty} \mathrm{e}^{-\int_{\tau}^{x}\left[i b+\lambda_{0}+\mu(s)\right] \mathrm{d} s} y_{4}(\tau) \mathrm{d}(\tau) \\
& p_{5}(x)=p_{5}(0) \mathrm{e}^{-\int_{0}^{x}\left[i b+\lambda_{1}+\lambda_{2}+r_{2}+\eta(s)\right] \mathrm{d} s}+\int_{0}^{\infty} \mathrm{e}^{-\int_{\tau}^{x}\left[i b+\lambda_{1}+\lambda_{2}+r_{2}+\eta(s)\right] \mathrm{d} s} y_{5}(\tau) \mathrm{d}(\tau) \\
& p_{6}(x)=p_{6}(0) \mathrm{e}^{-\int_{0}^{x}\left[i b+\lambda_{1}+\lambda_{2}+\eta(s)\right] \mathrm{d} s}+\int_{0}^{\infty} \mathrm{e}^{-\int_{\tau}^{x}\left[i b+\lambda_{1}+\lambda_{2}+\eta(s)\right] \mathrm{d} s} y_{6}(\tau) \mathrm{d}(\tau) \\
& p_{7}(x)=p_{7}(0) \mathrm{e}^{-\int_{0}^{x}\left[i b+\mu_{3}(s)\right] \mathrm{d} s}+\int_{0}^{\infty} \mathrm{e}^{-\int_{\tau}^{x}\left[i b+\mu_{3}(s)\right] \mathrm{d} s} y_{7}(\tau) \mathrm{d}(\tau) \\
& p_{8}(x)=p_{8}(0) \mathrm{e}^{-\int_{0}^{x}\left[i b+r_{2}+\mu_{1}(s)\right] \mathrm{d} s}+\int_{0}^{\infty} \mathrm{e}^{-\int_{\tau}^{x}\left[i b+r_{2}+\mu_{1}(s)\right] \mathrm{d} s} y_{8}(\tau) \mathrm{d}(\tau) \\
& p_{9}(x)=p_{9}(0) \mathrm{e}^{-\int_{0}^{x}\left[i b+r_{2}+\mu_{2}(s)\right] \mathrm{d} s}+\int_{0}^{\infty} \mathrm{e}^{-\int_{\tau}^{x}\left[i b+r_{2}+\mu_{2}(s)\right] \mathrm{d} s} y_{9}(\tau) \mathrm{d}(\tau) \\
& p_{10}(x)=p_{10}(0) \mathrm{e}^{-\int_{0}^{x}\left[i b+\lambda_{2}+\mu_{1}(s)\right] \mathrm{d} s}+\int_{0}^{\infty} \mathrm{e}^{-\int_{\tau}^{x}\left[i b+\lambda_{2}+\mu_{1}(s)\right] \mathrm{d} s} y_{10}(\tau) \mathrm{d}(\tau) \\
& p_{11}(x)=p_{11}(0) \mathrm{e}^{-\int_{0}^{x}\left[i b+\lambda_{1}+\mu_{2}(s)\right] \mathrm{d} s}+\int_{0}^{\infty} \mathrm{e}^{-\int_{\tau}^{x}\left[i b+\lambda_{1}+\mu_{2}(s)\right] \mathrm{d} s} y_{11}(\tau) \mathrm{d}(\tau) \\
& p_{12}(x)=p_{12}(0) \mathrm{e}^{-\int_{0}^{x}[i b+\beta(s)] \mathrm{d} s}+\int_{0}^{\infty} \mathrm{e}^{-\int_{\tau}^{x}[i b+\beta(s)] \mathrm{d} s} y_{12}(\tau) \mathrm{d}(\tau) \\
& p_{2}(0)=\lambda_{1} p_{0}, \quad p_{3}(0)=\lambda_{2} p_{0} \\
& p_{4}(0)=\lambda_{2} \int_{0}^{\infty} p_{2}(x) \mathrm{d} x+\lambda_{1} \int_{0}^{\infty} p_{3}(x) \mathrm{d} x \\
& p_{5}(0)=\lambda_{0} p_{0}+r_{1} p_{1}+\int_{0}^{\infty} \mu_{1}(x) p_{8}(x) \mathrm{d} x+\int_{0}^{\infty} \mu_{2} p_{9}(x) \mathrm{d} x \\
& p_{6}(0)=r_{2} \int_{0}^{\infty} p_{5}(x) \mathrm{d} x+\int_{0}^{\infty} \mu_{1} p_{10}(x) \mathrm{d} x+\int_{0}^{\infty} \mu_{2} p_{11}(x) \mathrm{d} x \\
& p_{7}(0)=\lambda_{3} p_{0}, \quad p_{8}(0)=\lambda_{1} \int_{0}^{\infty} p_{5}(x) \mathrm{d} x, \quad p_{9}(0)=\lambda_{2} \int_{0}^{\infty} p_{5}(x) \mathrm{d} x \\
& p_{10}(0)=\lambda_{1} \int_{0}^{\infty} p_{6}(x) \mathrm{d} x+r_{2} \int_{0}^{\infty} p_{8}(x) \mathrm{d} x
\end{aligned}
$$




$$
\begin{aligned}
p_{11}(0) & =\lambda_{2} \int_{0}^{\infty} p_{6}(x) \mathrm{d} x+r_{2} \int_{0}^{\infty} p_{9}(x) \mathrm{d} x \\
p_{12}(0) & =\lambda_{2} \int_{0}^{\infty} p_{10}(x) \mathrm{d} x+\lambda_{1} \int_{0}^{\infty} p_{11}(x) \mathrm{d} x
\end{aligned}
$$

Theorem 3 The system is exponentially stable.

Proof Let one rank projection $P$ be the associated spectral projection of the eigenvalue 0 since 0 is an algebraically simple eigenvalue of $\mathcal{A}$. Thus $X$ has the invariant decomposition

$$
X=X_{0}+Y, \quad X_{0}=P X, \quad Y=(I-P) X,
$$

in which $X_{0}$ is the eigenfunction subspace whose dimension is one. $Y$ is also Banach space equipped with the norm of $X$. Let $T_{1}(t)$ and $A_{1}$ be the restrictions of $T(t)$ and $A$ on $Y$ respectively. Obviously, we have $\sigma\left(A_{1}\right)=\sigma(A) \backslash\{0\}$. Now we will show that $s_{0}\left(A_{1}\right) \leq 0$. However, 0 is a regular point of $A_{1}$, so it is sufficient to show that

$$
\sup _{\gamma=i b,|b|>\delta>0, b \in R}\left\|R\left(\lambda, A_{1}\right)\right\|<+\infty .
$$

In fact, for $r=i b, b \in \mathbb{R}$ and each given $y \in X$, from Theorem 2 the equation $(r I-A) p=y$ has solution $p \in D(A)$ and $p_{i}(x)$ is given by (17) and (18). Thus we have

$$
\begin{aligned}
& |b| \int_{0}^{\infty}\left|p_{i}(x)\right| \mathrm{d} x \\
\leq & \int_{0}^{\infty}\left|p_{i}(0) r \mathrm{e}^{-\int_{0}^{x}\left[r+\gamma_{i}+h_{i} \xi\right] \mathrm{d} \xi}\right| \mathrm{d} x+|b| \int_{0}^{\infty}\left|\int_{0}^{\infty} y_{i}(\tau) \mathrm{e}^{-\int_{\tau}^{x}\left[r+\gamma_{i}+h_{i}(\xi) \mathrm{d}(\xi)\right]}\right| \mathrm{d} x \\
& |b| \int_{0}^{\infty}\left|p_{2+i}(x)\right| \mathrm{d} x \\
\leq & \int_{0}^{\infty}\left|p_{2+i}(0) r \mathrm{e}^{-\int_{0}^{x}\left[r+h_{i} \xi\right] \mathrm{d} \xi}\right| \mathrm{d} x+\int_{0}^{\infty}\left|p_{i}(0) r\left(\mathrm{e}^{-\int_{0}^{x}\left[r+h_{i} \xi\right] \mathrm{d} \xi}-r \mathrm{e}^{-\int_{0}^{x}\left[r+\gamma_{i}+h_{i} \xi\right] \mathrm{d} \xi}\right)\right| \mathrm{d} x \\
+ & \frac{|b|}{c} \int_{0}^{\infty} y_{2+i}(x) \mathrm{d} x+|b| \int_{0}^{\infty}\left|\int_{0}^{x} y_{i}(\tau)\left(\mathrm{e}^{-\int_{\tau}^{x}\left[r+h_{i}(\xi)\right] \mathrm{d} \xi}-\mathrm{e}^{-\int_{\tau}^{x}\left[r+\gamma_{i}+h_{i} \xi\right] \mathrm{d} \xi}\right) \mathrm{d} \tau\right| \mathrm{d} x
\end{aligned}
$$

From (13), we have the following inequality

$$
(|b|+\gamma)\left|p_{0}\right| \leq\left|y_{0}\right|+\sum_{i=1}^{2}\left|\int_{0}^{\infty} p_{i}(0) h_{i}(x) \mathrm{e}^{-\int_{0}^{\infty}\left(r+\gamma_{i}+h_{i}(\xi)\right) \mathrm{d} \xi} \mathrm{d} x\right|+\sum_{i=1}^{2} \int_{0}^{\infty}\left|y_{i}(x)\right| \mathrm{d} x
$$

Moreover, by the boundary conditions (14) (16) we have

$$
\begin{aligned}
\left|p_{1}(0)\right| \leq & \lambda_{1}\left|p_{0}\right|+\int_{0}^{\infty}\left|p_{2}(0)\left(1-r \mathrm{e}^{-\int_{0}^{x}\left[r+h_{2}(\xi)\right] \mathrm{d} \xi}-h_{2}(x) \mathrm{e}^{-\int_{0}^{x}\left[r+h_{2}(\xi)\right] \mathrm{d} \xi}\right)\right| \mathrm{d} x \\
& +\int_{0}^{\infty}\left|p_{4}(0)\left(1-r \mathrm{e}^{-\int_{0}^{x}\left[r+h_{2}(\xi)\right] \mathrm{d} \xi}\right)\right| \mathrm{d} x+2 \int_{0}^{\infty}\left|y_{2}(x)\right| \mathrm{d} x+\int_{0}^{\infty}\left|y_{4}(x)\right| \mathrm{d} x \\
\left|p_{2}(0)\right| \leq & \lambda_{2}\left|p_{0}\right|+\int_{0}^{\infty}\left|p_{1}(0)\left(1-r \mathrm{e}^{-\int_{0}^{x}\left[r+h_{1}(\xi)\right] \mathrm{d} \xi}-h_{1}(x) \mathrm{e}^{-\int_{0}^{x}\left[r+h_{1}(\xi)\right] \mathrm{d} \xi}\right)\right| \mathrm{d} x \\
& +\int_{0}^{\infty}\left|p_{3}(0)\left(1-r \mathrm{e}^{-\int_{0}^{x}\left[r+h_{1}(\xi)\right] \mathrm{d} \xi}\right)\right| \mathrm{d} x+2 \int_{0}^{\infty}\left|y_{1}(x)\right| \mathrm{d} x+\int_{0}^{\infty}\left|y_{3}(x)\right| \mathrm{d} x
\end{aligned}
$$

If adding (43) (47) together, we would have the following estimate

$$
|b|\left(\left|p_{0}\right|+\sum_{i=1}^{4} \int_{0}^{\infty}\left|p_{i}(x)\right| \mathrm{d} x\right) \leq\left|y_{0}\right|+\left(1+\frac{|b|}{c}\right) \sum_{i=1}^{4} \int_{0}^{\infty}\left|y_{i}(x)\right| \mathrm{d} x+2 \sum_{i=1}^{2} \int_{0}^{\infty}\left|y_{i}(x)\right| \mathrm{d} x
$$


By the definition of the norm on the space $X$ and (48), we obtain that

$$
\left\|R\left(r I-A_{1}\right)\right\| \leq \frac{1}{|b|}\left(2+\frac{|b|}{c}\right) .
$$

Therefore we know that,

$$
\lim _{b \rightarrow+\infty}\left\|R\left(r I-A_{1}\right)\right\|=\frac{1}{c}
$$

and

$$
\sup _{r=i b,|b|>\delta>0, b \in R}\left\|R\left(r I-A_{1}\right)\right\|<+\infty .
$$

From the definition of $s_{0}(A)$ and Lemma 2, we obtain that $s_{0}\left(A_{1}\right) \leq 0$ and $\omega_{1}\left(T_{1}\right)<0$ due to $\omega_{1}\left(T_{1}\right)<s_{0}\left(A_{1}\right)$. However, by [14] we know semigroups $T(t)$ is exponentially stable if and only if $\omega_{1}(T)<0$. Thus we complete the proof of Theorem 3 .

In fact, the exponential stability of the repairable system was firstly given in [14]. You can refer to the reference [14] in the modified version for more information. To show the effectiveness of the reliability of the repair system, we include this part again in this note. The method is mainly employed from [3].

\section{Some steady-state reliability indices}

According to [2],

$$
p_{0}(t)+\sum_{i=2}^{6} \int_{0}^{\infty} p_{i}(x, t) \mathrm{d} x+\sum_{i=8}^{11} \int_{0}^{\infty} p_{i}(x, t) \mathrm{d} x
$$

and

$$
A V=\lim _{t \rightarrow \infty}\left(p_{0}(t)+\sum_{i=2}^{6} \int_{0}^{\infty} p_{i}(x, t) \mathrm{d} x+\sum_{i=8}^{11} \int_{0}^{\infty} p_{i}(x, t) \mathrm{d} x\right)
$$

are the instantaneous availability and the stationary availability of the repairable system, respectively. The availability of the system is one of the most important reliability indices and engineers are especially interested in the steady-state availability. Now we will obtain the stationary availability of the system with another method different from the traditional one. For convenience, set

$$
\begin{aligned}
& f_{2}(x)=\mathrm{e}^{-\int_{0}^{x}\left[\mu_{1}(s)+\lambda_{2}\right] \mathrm{d} s}, \quad a_{2}=\int_{0}^{\infty} \mu_{1}(x) f_{2}(x) \mathrm{d} x, \quad b_{2}=\int_{0}^{\infty} f_{2}(x) \mathrm{d} x, \\
& f_{3}(x)=\mathrm{e}^{-\int_{0}^{x}\left[\mu_{2}(s)+\lambda_{1}\right] \mathrm{d} s}, \quad a_{3}=\int_{0}^{\infty} \mu_{2}(x) f_{3}(x) \mathrm{d} x, \quad b_{2}=\int_{0}^{\infty} f_{3}(x) x, \\
& f_{4}(x)=\mathrm{e}^{-\int_{0}^{x}\left[\mu(s)+\lambda_{0}\right] \mathrm{d} s}, \quad a_{4}=\int_{0}^{\infty} \mu(x) f_{4}(x) \mathrm{d} x, \quad b_{4}=\int_{0}^{\infty} f_{4}(x) \mathrm{d} x, \\
& f_{5}(x)=\mathrm{e}^{-\int_{0}^{x}\left[\eta(s)+\lambda_{1}+\lambda_{2}+r_{2}\right] \mathrm{d} s}, \quad a_{5}=\int_{0}^{\infty} \eta(x) f_{5}(x) \mathrm{d} x, \quad b_{5}=\int_{0}^{\infty} f_{5}(x) \mathrm{d} x, \\
& f_{6}(x)=\mathrm{e}^{-\int_{0}^{x}\left[\eta(s)+\lambda_{1}+\lambda_{2}\right] \mathrm{d} s}, \quad a_{6}=\int_{0}^{\infty} \eta(x) f_{6}(x) \mathrm{d} x, \quad b_{6}=\int_{0}^{\infty} f_{6}(x) \mathrm{d} x, \\
& f_{7}(x)=\mathrm{e}^{-\int_{0}^{x} \mu_{3}(s) \mathrm{d} s}, \quad b_{7}=\int_{0}^{\infty} f_{7}(x) \mathrm{d} x, \\
& f_{8}(x)=\mathrm{e}^{-\int_{0}^{x}\left[\mu_{1}(s)+r_{2}\right] \mathrm{d} s}, \quad a_{8}=\int_{0}^{\infty} \mu_{1}(x) f_{8}(x) \mathrm{d} x, \quad b_{8}=\int_{0}^{\infty} f_{8}(x) \mathrm{d} x,
\end{aligned}
$$




$$
\begin{aligned}
f_{9}(x) & =\mathrm{e}^{-\int_{0}^{x}\left[\mu_{2}(s)+r_{2}\right] \mathrm{d} s}, & a_{9} & =\int_{0}^{\infty} \mu_{2}(x) f_{9}(x) \mathrm{d} x, \\
f_{12}(x) & =\mathrm{e}^{-\int_{0}^{x} \beta(s) \mathrm{d} s}, & b_{12} & =\int_{0}^{\infty} f_{12}(x) \mathrm{d} x .
\end{aligned}
$$

Theorem 4 The stationary availability of the system is

$$
A V=\frac{1+\sum_{i=4}^{6} b_{i} c_{i}+\sum_{i=8}^{9} b_{i} c_{i}+b_{2}\left(c_{10}+\lambda_{1}\right)+b_{3}\left(c_{11}+\lambda_{2}\right)}{1+c_{1}+\sum_{i=4}^{6} b_{i} c_{i}+\lambda_{3} b_{7}+\sum_{i=8}^{9} b_{i} c_{i}+b_{2}\left(c_{10}+\lambda_{1}\right)+b_{3}\left(c_{11}+\lambda_{2}\right)+b_{12} c_{12}}
$$

in which

$$
\begin{aligned}
& c_{1}=r_{1}^{-1} \lambda_{0} \lambda_{1} \lambda_{2} b_{4}\left(b_{2}+b_{3}\right), \quad c_{4}=\lambda_{1} \lambda_{2}\left(b_{2}+b_{3}\right), \\
& c_{5}=\left[1-b_{5}\left(\lambda_{1} a_{8}+\lambda_{2} a_{9}\right)\right]^{-1}\left[\lambda_{0}+\lambda_{0} \lambda_{1} \lambda_{2} b_{4}\left(b_{2}+b_{3}\right)\right], \\
& c_{8}=\lambda_{1} b_{5}\left[1-b_{5}\left(\lambda_{1} a_{8}+\lambda_{2} a_{9}\right)\right]^{-1}\left[\lambda_{0}+\lambda_{0} \lambda_{1} \lambda_{2} b_{4}\left(b_{2}+b_{3}\right)\right], \\
& c_{9}=\lambda_{2} b_{5}\left[1-b_{5}\left(\lambda_{1} a_{8}+\lambda_{2} a_{9}\right)\right]^{-1}\left[\lambda_{0}+\lambda_{0} \lambda_{1} \lambda_{2} b_{4}\left(b_{2}+b_{3}\right)\right], \\
& c_{6}=r_{2}\left(b_{5} c_{5}+a_{2} b_{8} c_{8}+a_{3} b_{9} c_{9}\right)\left[1-b_{6}\left(\lambda_{1} a_{2}+\lambda_{2} a_{3}\right)\right]^{-1}, \\
& c_{10}=\lambda_{1} b_{6} c_{6}+r_{2} b_{8} c_{8}, c_{11}=\lambda_{2} b_{6} c_{6}+r_{2} b_{9} c_{9}, c_{12}=\lambda_{2} b_{2} c_{10}+\lambda_{1} b_{3} c_{11},
\end{aligned}
$$

Proof In order to compute the stationary availability of the discussed system, we need to obtain the formulation of the positive unit eigenfunction $\widetilde{p}$ corresponding to eigenvalue 0 of the operator $\mathcal{A}$. Now we complete this job. In fact, $\mathcal{A} p=0$ is the following set of equations:

$$
\begin{aligned}
p_{2}^{\prime}(x) & =\left[\mu_{1}(x)+\lambda_{2}\right] p_{2}(x), & p_{3}^{\prime}(x) & =\left[\mu_{2}(x)+\lambda_{1}\right] p_{3}(x), \\
p_{4}^{\prime}(x) & =\left[\mu(x)+\lambda_{0}\right] p_{4}(x), & p_{5}^{\prime}(x) & =\left[\eta(x)+\lambda_{1}+\lambda_{2}+r_{2}\right] p_{5}(x), \\
p_{6}^{\prime}(x) & =\left[\eta(x)+\lambda_{1}+\lambda_{2}\right] p_{6}(x), & p_{7}^{\prime}(x) & =\mu_{3}(x) p_{7}(x), \\
p_{8}^{\prime}(x) & =\left[\mu_{1}(x)+r_{2}\right] p_{8}(x), & p_{9}^{\prime}(x) & =\left[\mu_{2}(x)+r_{2}\right] p_{9}(x), \\
p_{10}^{\prime}(x) & =\left[\mu_{1}(x)+\lambda_{2}\right] p_{10}(x), & p_{11}^{\prime}(x) & =\left[\mu_{2}(x)+\lambda_{1}\right] p_{11}(x)
\end{aligned}
$$

with the initial conditions:

$$
\begin{aligned}
r_{1} p_{1} & =\lambda_{0} \int_{0}^{\infty} p_{4}(x) \mathrm{d} x, \quad p_{2}(0)=\lambda_{1} p_{0}, \quad p_{0}(0)=\lambda_{2} p_{0} \\
p_{4}(0) & =\lambda_{2} \int_{0}^{\infty} p_{2}(x) \mathrm{d} x+\lambda_{1} \int_{0}^{\infty} p_{3}(x) \mathrm{d} x \\
p_{5}(0) & =\lambda_{0} p_{0}+r_{1} p_{1}+\int_{0}^{\infty} \mu_{1}(x) p_{8}(x) \mathrm{d} x+\int_{0}^{\infty} \mu_{2}(x) p_{9}(x) \mathrm{d} x \\
p_{6}(0) & =r_{2} \int_{0}^{\infty} p_{5}(x) \mathrm{d} x+\int_{0}^{\infty} \mu_{1}(x) p_{10}(x) \mathrm{d} x+\int_{0}^{\infty} \mu_{2}(x) p_{11}(x) \mathrm{d} x \\
p_{7}(0) & =\lambda_{3} p_{0}, \quad p_{8}(0)=\lambda_{1} \int_{0}^{\infty} p_{5}(x) \mathrm{d} x, \quad p_{8}(0)=\lambda_{2} \int_{0}^{\infty} p_{5}(x) \mathrm{d} x \\
p_{10}(0) & =\lambda_{1} \int_{0}^{\infty} p_{6}(x) \mathrm{d} x+r_{2} \int_{0}^{\infty} p_{8}(x) \mathrm{d} x \\
p_{11}(0) & =\lambda_{2} \int_{0}^{\infty} p_{6}(x) \mathrm{d} x+r_{2} \int_{0}^{\infty} p_{9}(x) \mathrm{d} x \\
p_{12}(0) & =\lambda_{2} \int_{0}^{\infty} p_{10}(x) \mathrm{d} x+\lambda_{1} \int_{0}^{\infty} p_{11}(x) \mathrm{d} x
\end{aligned}
$$


Solving equations (50), we have

$$
\begin{aligned}
p_{i}(x) & =p_{i}(0) f_{i}(x), i=2,3, \cdots, 9, \quad p_{10}(x)=p_{10}(0) f_{2}(x) \\
p_{11}(x) & =p_{11}(0) f_{2}(x), \quad p_{12}(x)=p_{12}(0) f_{12}(x)
\end{aligned}
$$

By the Theorem 4.1 of [1], because 0 is a simple eigenvalue of $\mathcal{A}$ and there exists a corresponding positive eigenvector, we can set $p_{0}=1$. With the help of

$$
\int_{0}^{\infty} u_{i}(x) \mathrm{e}^{-\int_{0}^{x} u_{i}(\tau) \mathrm{d} \tau} \mathrm{d} x=1
$$

and the notations of $c_{i}$, it is easy to know that

$$
p_{1}=c_{1}, p_{2}(0)=\lambda_{1}, p_{3}(0)=\lambda_{2}, p_{i}(0)=c_{i}(i=4,5,6), p_{7}(0)=\lambda_{3},
$$

and

$$
p_{j}(0)=c_{j}(j=8,9, \cdots, 12)
$$

Thus,

$$
\begin{array}{r}
P=\left(1, c_{1}, \lambda_{1} f_{2}(x), \lambda_{2} f_{3}(x), c_{4} f_{4}(x), c_{5} f_{5}(x), c_{6} f_{6}(x), \lambda_{3} f_{7}(x),\right. \\
\left.c_{8} f_{8}(x), c_{9} f_{9}(x), c_{10} f_{10}(x), c_{11} f_{11}(x), c_{12} f_{12}(x)\right)
\end{array}
$$

is the positive eigenfunction corresponding to eigenvalue 0 of the operator $\mathcal{A}$ and $\widetilde{p}=\|P\|^{-1} P$. Therefore, we can get the expression $A V=1-\left.\left(c_{1}+b_{7} c_{7}+c_{12} c_{12}\right)\|P\|\right|^{-1}$ by the definition of the stationary availability, that is the formula (49) due to

$$
\|P\|=1+c_{1}+\sum_{i=4}^{6} b_{i} c_{i}+\lambda_{3} b_{+} \sum_{i=8}^{9} b_{i} c_{i}+b_{2}\left(c_{10}+\lambda_{1}\right)+b_{3}\left(c_{11}+\lambda_{2}\right)+b_{12} c_{12} .
$$

We complete the proof of the Theorem 4. With the help of Theorem 4, we are able to calculate the mean up-time of the redundant system.

Theorem 5 The mean up-time (MUT) of the redundant system is equal to

$$
M U T=\frac{1+\sum_{i=4}^{6} b_{i} c_{i}+\lambda_{3} b_{+} \sum_{i=8}^{9} b_{i} c_{i}+b_{2}\left(c_{10}+\lambda_{1}\right)+b_{3}\left(c_{11}+\lambda_{2}\right)}{\lambda_{0}\left(1+b_{4} c_{4}\right)+\lambda_{2} b_{2}\left(c_{10}+\lambda_{1}\right)+\lambda_{1} b_{3}\left(c_{11}+\lambda_{2}\right)+\left(\lambda_{1}+\lambda_{1}\right)\left(b_{5} c_{5}+b_{6} c_{6}\right)+\lambda_{3}^{2} b_{7}} .
$$

Proof Let $M$ be the rate of failures of the system, then

$$
M=\|p\|^{-1}\left[\lambda_{0}\left(1+p_{4}\right)+\lambda_{2}\left(p_{2}+p_{10}\right)+\lambda_{1}\left(p_{3}+p_{11}\right)+\left(\lambda_{1}+\lambda_{1}\right)\left(p_{5}+p_{6}\right)+\lambda_{3} b_{7}\right] .
$$

From the proof of the Theorem 4, we know $p_{2}=\lambda_{1} b_{2}, p_{3}=\lambda_{2} b_{3}, p_{7}=\lambda_{3} b_{2}, p_{i}=c_{i} b_{i}, i=$ $4,5,6, p_{10}=c_{10} b_{2}$, and $p_{11}=c_{11} b_{3}$. However, the mean up-time $(M U T)$ of the redundant system is $\frac{A V}{M}$. Computing directly leads to the formulation of $M U T$.

Corollary 1 The stationary availability and MUT of the system without standby are

$$
A V^{\prime}=\frac{1}{1+\lambda_{3} b_{7}+\lambda_{0} b_{12}}, \quad M U T^{\prime}=\frac{1}{\lambda_{0}+\lambda_{3}^{2} b_{7}} .
$$

\section{Acknowledgments}

The authors would like to thank the referees for their helpful suggestions and comments. 


\section{References}

[1] Itoi T, Kodama M, Nishida T. Behavior of a two correlated units redundant system with many types of failure. Microeletronics Reliability, 1978, 17: 269-271.

[2] Cao J H, Cheng K. Introduction to reliability mathematics. Science Press, Beijing, China, 1986.

[3] Wang W L, Xu G Q. Well-posedness and stability of solution of a series system with repair facility. Applied Mathematics A Journal of Chinese Universities (Ser A), 2007, 22: 474-482.

[4] Wang W L, Xu G Q. Stability analysis of a complex standby system with constant waiting and different repairman criteria incorporating environmental failure. Applied Mathematical Modelling, 2009, 33: 724743.

[5] Hu W W, Shen Z F, Xin Y H, et al. Exponential stability of a repairable system with imperfect switching mechanism. Asymptotic Analysis, 2007, 54: 93-102.

[6] Shen Z F, Hu X X, Fan W F, et al. Exponential asymptotic property of a parallel repairable system with warm standby under common-cause failure. Journal of Mathematical Analysis and Applications, 2008, 341: 457-466.

[7] Guo L N, Xu H B, Gao C, et al. Stability analysis of a new kind series system. IMA Journal of Applied Mathematics, 2010, 75: 439-460.

[8] Gupur G, Li X Z, Zhu G T. Functional analysis method in queueing theory. Hertfordshire, Research Information Ltd: United Kingdom, 2001.

[9] Wang J T. An $M / G / 1$ queue with second potional service and server breakdowns. Computers and Mathematics with Applications, 2004, 47: 1713-1723.

[10] Haji A, Radl A. A semigroup approach to queueing systems. Semigroup Forum, 2007, 75: 609-623.

[11] Pazy A. Semigroup of linear operators and application to partial differential equations. Spring-verlag, New York, 1983.

[12] Engel K J, Nagel R. A short course on operator semigroups. Springer, 2006.

[13] Dunford N, Schwartz J T. Linear operators I. Wiley, New York, 1958.

[14] Zheng F, Yang J Y, Zhu G T. Exponential stability of a complex standby system. Advanced Mathematics, 2012, 41(6): 713-722.

[15] Dyson J, Villella-Bressan R, Webb G F. Asynchronous exponential growth in an age structured population of proliferating and quiescent cells. Mathematical Biosciences, 2002, 177-178: 73-83.

[16] Arino O, Bertuzzi A, Gandolfi A, et al. The asynchronous exponential growth property in a model for the kinetic heterogeneity of tumour cell populations. Journal of Mathematical Analysis and Applications, 2005, 302: $521-542$ 